\title{
A Long-Term Follow-up of Pontine Hemorrhage With Hearing Loss
}

\author{
Seung Ki Kim, MD, Ae Ryoung Kim, MD, Joon Yeop Kim, MD, Deog Young Kim, MD, PhD
}

Department of Rehabilitation Medicine and Research Institute of Rehabilitation Medicine, Yonsei University College of Medicine, Seoul, Korea

\begin{abstract}
A pontine intracranial hemorrhage (ICH) evokes several neurological symptoms, due to the various nuclei and nerve fibers; however, hearing loss from a pontine ICH is rare. We have experienced a non-traumatic pontine ICH patient, with hearing loss. A 43-year-old male patient had a massive pontine hemorrhage; his brain magnetic resonance imaging revealed the hemorrhage on the bilateral dorsal pons, with the involvement of the trapezoid body. Also, profound hearing loss on the pure-tone audiogram and abnormal brainstem auditory evoked potential were noticed. Fifty-two months of long-term follow-up did not reveal any definite improvement on the patient's hearing ability.
\end{abstract}

Keywords Hearing loss, Intracranial hemorrhages, Pons

\section{INTRODUCTION}

Non-traumatic pontine hemorrhages occur in about $10 \%$ of all non-traumatic intracranial hemorrhage (ICH) cases [1]. Due to the various nuclei and nerve fibers concentrated in the pons, several neurological symptoms specific to the site of the lesion can be observed in the patients with the pontine lesions [2]. A large central hematoma usually occurs between the basis pontine and the tegmentum, and expands to the rostral midbrain and the 4 th ventricle, developing into a loss of consciousness,

Received July 24, 2014; Accepted September 15, 2014

Corresponding author: Deog Young Kim

Department of Rehabilitation Medicine and Research Institute of Rehabilitation Medicine, Yonsei University College of Medicine, 50 Yonsei-ro, Seodaemun-gu, Seoul 120-749, Korea

Tel: +82-2-2228-3714, Fax: +82-2-363-2795, E-mail: kimdy@yuhs.ac

(c) This is an open-access article distributed under the terms of the Creative Commons Attribution Non-Commercial License (http://creativecommons. org/licenses/by-nc/4.0) which permits unrestricted noncommercial use, distribution, and reproduction in any medium, provided the original work is properly cited.

Copyright $\odot 2015$ by Korean Academy of Rehabilitation Medicine quadriplegia, ataxia, pinpoint pupils, limited horizontal gaze movement, and ocular bobbing [1]. Despite the hearing conduction pathway, which passes through the cochlear nuclei in the pons, hearing loss from a pontine hemorrhage is very rare [3].

We are reporting on a case of a patient with hearing loss, with a pontine ICH, with 4 years' long-term followup.

\section{CASE REPORT}

A 43-year-old male had suffered a headache for few days, and was admitted to an emergency department due to a change in his mental status. On arrival, a brain computed tomography (CT) showed a massive hematoma in the dorsal pons (Fig. 1). He took conservative treatments, without operation. Two months later, the patient's caregiver complained of a communication problem, stating that the man could not seem to understand the instructions from his therapist and caregiver. There was no definite lesion other than the fluid collection in both sides of 

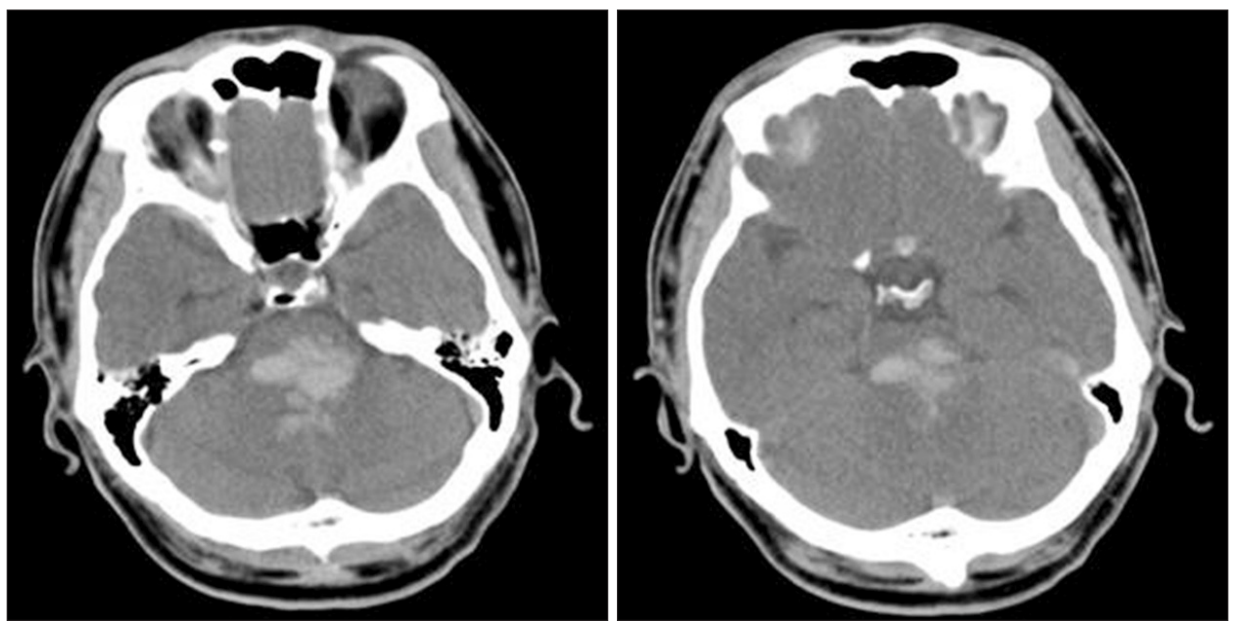

Fig. 1. The initial brain computed tomography.
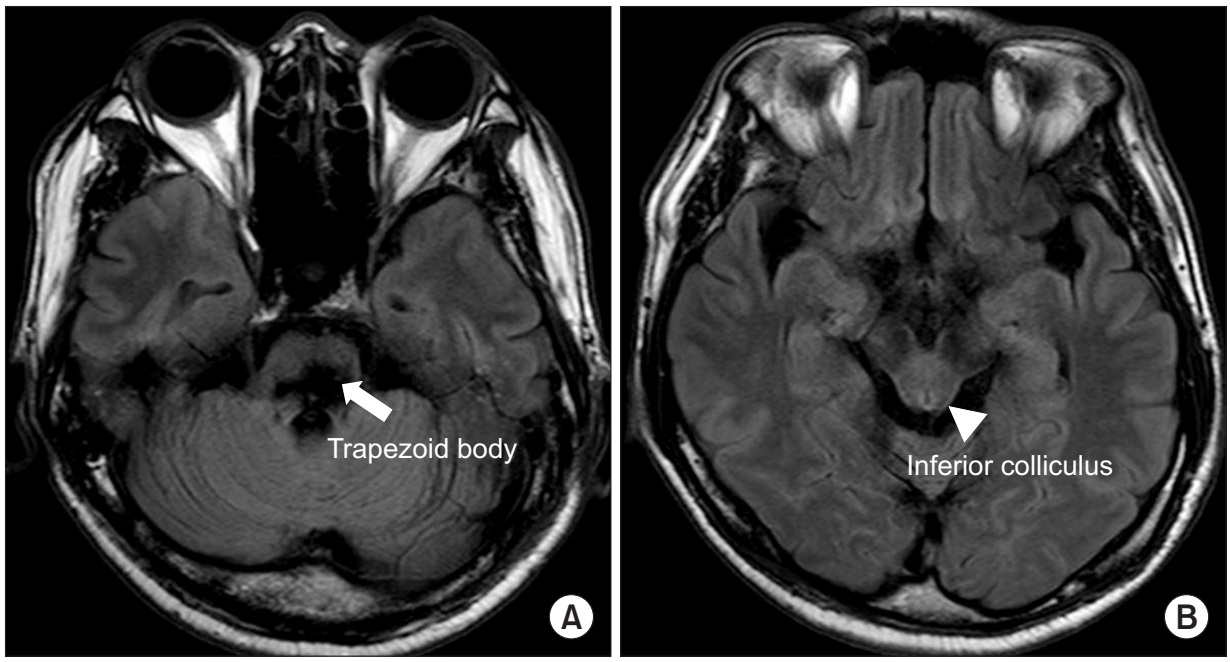

Fig. 2. The findings of the magnetic resonance imaging T2 fluid attenuated inversion recovery (FLAIR) at 3 months from the onset. (A) Pons. (B) Midbrain.

the mastoid air cells, on the temporal bone CT, and the brainstem auditory evoked potential (BAEP) test revealed prolonged latencies of III and V (from both sides), which were suspected to be a central conduction defect. Three months after the onset, the patient was transferred to our department for comprehensive rehabilitative management. At that time, a brain magnetic resonance imaging (MRI) showed an old hemorrhage in the bilateral dorsal pons (at the level of the vestibulocochlear nerves) which did not involve the inferior colliculus at the midbrain but rather the trapezoid body (Fig. 2). Severe ataxia, vertical nystagmus, limited horizontal eye movements, left facial palsy and paresthesia, and hearing loss on both sides were present, and the limb muscles were measured as being grade 4, grossly. His Mini-Mental State Examination (MMSE) was 20 points; after that, it gradully improved. At the 6-month follow-up, the MMSE was 28 points, and at 12 months, it was 29 points. After that, it remained without significant changes.

He could not detect individual words or sentences without a hearing aid. The pure tone audiogram (PTA), at 3 months from the onset, showed an average of $100 \mathrm{~dB}$ on the right side, and $99 \mathrm{~dB}$ on the left side; only I waves were shown on both sides in the BAEP. He was only able to respond to loud conversation, with the use of a hearing aid (Categories of Auditory Performance [CAP]-2); he could detect only parts of nasal vowel sounds $(a, i)$ in the low-frequency range and fricative sounds $(\mathrm{s}, \mathrm{sh})$ in the high-frequency range accurately in the Ling six sound test.

Afterward, speech therapy that focused on lip reading and the ability to distinguish speech sound were 


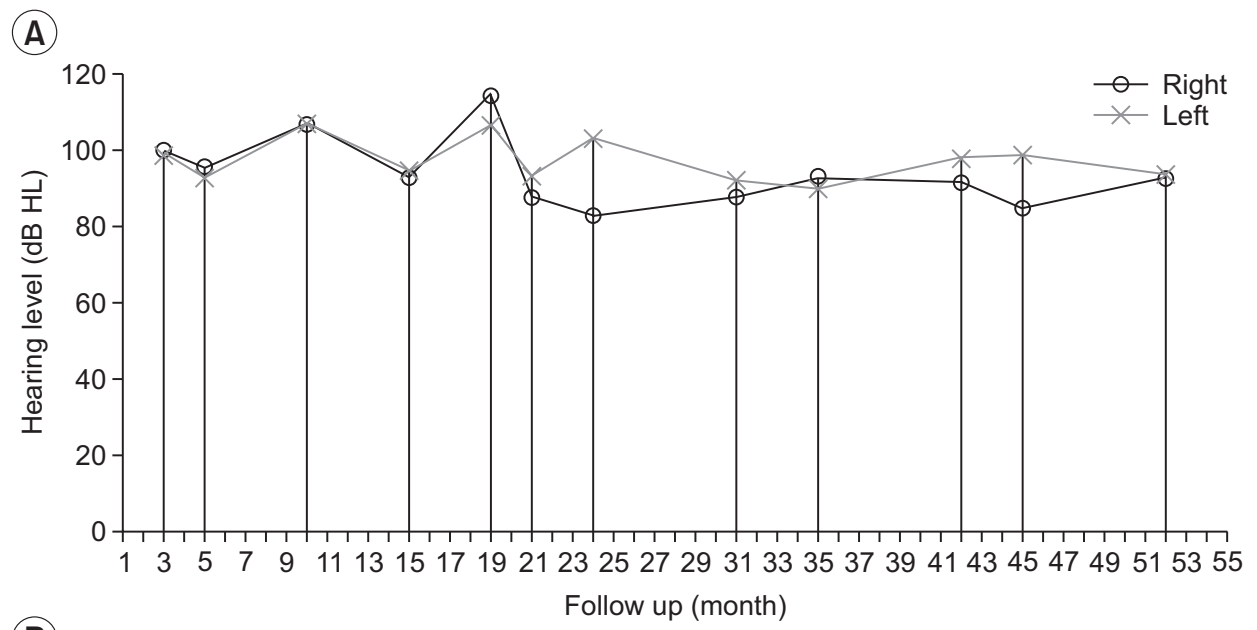

(B)
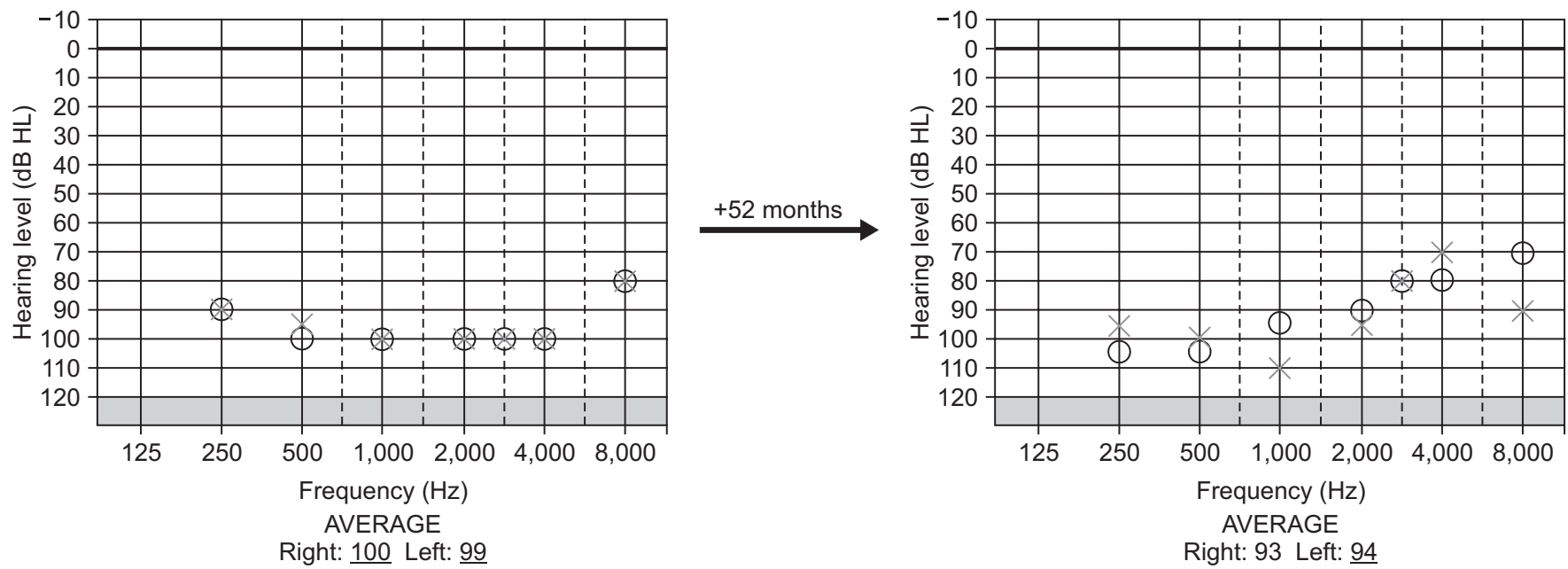

Fig. 3. (A) Changes of the mean pure tone audiogram (PTA) during the 52 months follow-up. (B) A comparison of the PTA according to the stimulus frequency between at 3 months and 52 months from the onset.

performed 2-3 times every week. The PTA, at 52 months from the onset did not show definite improvement (93 $\mathrm{dB}$ from the right side and $94 \mathrm{~dB}$ from the left side) compared to the previous PTAs, with the exception of the 10-20 dB level of improvement from the $2-8 \mathrm{kHz}$ section (Fig. 3). The patient's hearing loss did not change without a hearing aid, but he could confirm $80 \%$ of the 1st and 2nd syllables, and $56 \%$ of a sentence. In addition, he was able to identify environmental sounds with a hearing aid (CAP-3) and, on the Ling six sound test, the patient could consistently detect fricative sounds ( $\mathrm{sh}$ ) belonging to the high-frequency range.

The subject was able to maintain a rounded-back sitting posture, with severe ataxic movements (International Cooperative Ataxia Rating Scale [ICARS] score 82), and was in a totally dependent state (Modified Barthel Index
[MBI] score 5) at 3 months from the onset. He underwent physical and occupational therapy, which focused on strengthening the core muscles and training designed to improve one's balance while sitting. At 6 months from the onset, the ICARS showed a definite improvement, from 82 to 52 points; after that, it remained without definite changes. Left hypertrophic olivary degeneration (HOD) was observed on the follow-up brain MRI (Fig. 4). At 18 months from the onset, ataxia was seen to have gotten worse (ICARS score 61); a bilateral HOD and brain atrophy were shown from the T2 fluid attenuated inversion recovery (FLAIR), as compared to previous MRIs (Fig. 4). The ICARS scores did not change definitively until 48 months from onset (Table 1). Only his sitting balance was improved (from poor to good) without no definite changes in his standing and walking ability; the subject 

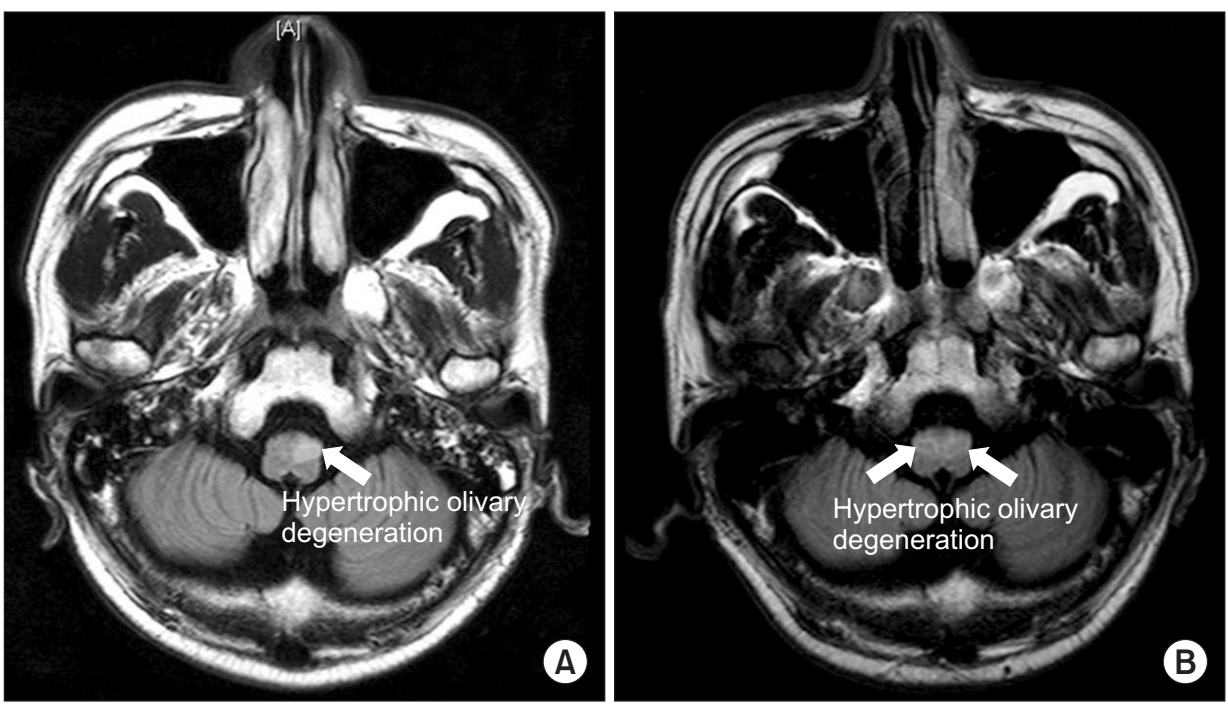

Fig. 4. Magnetic resonance imaging T2 fluid attenuated inversion recovery (FLAIR) at 6 months (A) and 18 months (B) from the onset.

Table 1. The changes of the International Cooperative Ataxia Rating Scale (ICARS)

\begin{tabular}{|c|c|c|c|c|c|c|c|c|}
\hline & $3 \mathrm{mo}$ & $4 \mathrm{mo}$ & $6 \mathrm{mo}$ & $12 \mathrm{mo}$ & $18 \mathrm{mo}$ & $24 \mathrm{mo}$ & $36 \mathrm{mo}$ & $48 \mathrm{mo}$ \\
\hline Postural and gait disturbance & 33 & 31 & 23 & 30 & 30 & 29 & 29 & 29 \\
\hline Limb ataxia & 36 & 29 & 22 & 17 & 24 & 26 & 27 & 26 \\
\hline Dysarthria & 8 & 6 & 4 & 6 & 6 & 6 & 6 & 6 \\
\hline Oculomotor disorders & 5 & 5 & 3 & 2 & 1 & 0 & 0 & 0 \\
\hline Total & 82 & 71 & 52 & 55 & 61 & 61 & 62 & 61 \\
\hline
\end{tabular}

remained totally dependent (MBI score 10).

\section{DISCUSSION}

Hearing loss after a stroke often develops in anterior inferior cerebellar artery infarction patients [4], but hearing loss after a pontine hemorrhage is rare [2,5-8]. The nerve fibers of the hearing-conducting pathway run bilaterally, from the cochlear nuclei to the inferior colliculus nuclei, through the lateral lemniscus. The second neuron projects impulses centrally along a number of different pathways, including crossing the midline within the trapezoid bodies. Not all auditory fibers cross the midline within the brainstem; part of the pathway remains ipsilateral. As a consequence, each side of the auditory area in the temporal cortex receives a signal from both cochlear nuclei. As such, it is rare for an injury to the second neuron of the hearing-conducting pathway to cause bilateral hearing loss.

Few researchers have reported hearing loss from a midline pontine tegmental hemorrhage [6], from a massive dorsal pontine hemorrhage [2], and from a ventral pontine hemorrhage [7]. The patient in this case study had a massive hemorrhage at the bilateral dorsal pons, which was similar to the study of Nakane et al. [2].

In regard to the mechanism of deafness after a pontine hemorrhage, Egan et al. [6] suggested the deactivation of the ventral acoustic striae decussating in the trapezoid body, which carries the majority of the neural signals responsible for hearing and speech perception. Nakane et al. [2] suggested a conduction deficit in the cochlear nuclei and the trapezoid body. Smart et al. [7] stated that the involvement of the inferior colliculus may cause hearing loss.

In this case, a massive hematoma was located at the bilateral dorsal pons, which extended widely in the lower to upper pons and, according to the MRI, the trapezoid body and the cochlear nuclei were intruded upon at the level of vestibulocochlear nerves; however, since the inferior colliculus was not intruded upon, as per the previous reports $[2,6]$. Considering these findings, we assumed that a massive hemorrhage, which was located in both 
the cochlear nuclei and the trapezoid bodies, caused a conduction deficit in the hearing nerve-tract, thus resulting in the bilateral hearing loss observed in this case. This is in line with the initial findings of the BAEP, which showed intact I waves and bilateral prolonged latencies of waves III and V. Wave III relates to structures in the pontomedullary region, and wave $\mathrm{V}$ reflects the function in the upper pons and low midbrain. In an animal model [9], the destruction of the trapezoid body leads to the disappearance of waves III to V. Since the BAEP showed only I waves at 3 months from the onset, it was assumed that the result of this was the progression of degeneration as time passed. The prognosis was still unknown and was controversial. During the over 4 years of follow-up in this case, the PTA did not show any changes from the initial, and word identification (in high pitch tones) was improved only slightly with a hearing aid.

Egan et al. [6] and Goyal et al. [8] reported a case of bilateral hearing loss with a normal BAEP latency at the initial, with almost complete recovery. Nakane et al. [2] reported that hearing loss, with only I waves on both BAEP after pontine hemorrhage meant the patient could only understand certain words at 6 months from the onset. In addition, Kim et al. [4] reported that about $65 \%$ of their vertebrobasilar ischemic stroke patients, with hearing loss, showed partial or complete hearing recovery after one year. In contrast to the cases above, our case showed signs of prolonged latencies of waves III and V, at the initial BAEP. Moreover, considering the signs that showed no response at 3 months from the onset, and profound hearing loss above $90 \mathrm{~dB}$ at the initial PTA, it can be assumed that this case includes more severe damage than the cases mentioned above. An abnormal BAEP at the initial may predict a poor prognosis. In this case, more severe damage (with no response of the BAEP at 3 months from the onset) did not show a good recovery.

Our finding that recognition of words showed greater improvement with high pitch tones, compared to low pitch tones, is in line with Nakane et al.'s report [2] that the hearing-conducting pathway of a high pitch tone area is positioned in a more outer portion compared to a low pitch tone area; then, hemorrhages and edemas may involve the pathway of high pitch tone area less.

The patient's ataxic movements improved until 6 months from onset, but then worsened. This may be related to the HOD, which was not observed at 3 months; this usually occurs when the lesion involves the dentatorubro-olivary pathway. A pontine hemorrhage frequently invades the ipsilateral central tegmental tract, the contralateral superior cerebellar peduncle, or the dentate nucleus [10].

This case reported improvement in the ataxia, but not in the hearing loss, through the long-term follow-up for a pontine hemorrhage patient. As shown in this case, when initially assessing pontine hemorrhage patients with altered mental status, hearing loss may be considered through the BAEP and PTA. An abnormal BAEP and PTA may predict a poor prognosis of hearing loss. Further studies will be needed, which can reveal the mechanism and prognosis of hearing loss in a pontine hemorrhage.

\section{CONFLICT OF INTEREST}

No potential conflict of interest relevant to this article was reported.

\section{REFERENCES}

1. Moncayo J. Pontine infarcts and hemorrhages. Front Neurol Neurosci 2012;30:162-5.

2. Nakane H, Sugimori H, Wakugawa $Y$, Hayashi R, Ibayashi S, Iida M. A case of hearing loss and quadriplegia after a pontine hemorrhage. J Neurol Sci 2006;241:91-4.

3. Schick B, Brors D, Koch O, Schafers M, Kahle G. Magnetic resonance imaging in patients with sudden hearing loss, tinnitus and vertigo. Otol Neurotol 2001;22:808-12.

4. Kim HA, Lee BC, Hong JH, Yeo CK, Yi HA, Lee H. Longterm prognosis for hearing recovery in stroke patients presenting vertigo and acute hearing loss. J Neurol Sci 2014;339:176-82.

5. Cohen M, Luxon L, Rudge P. Auditory deficits and hearing loss associated with focal brainstem haemorrhage. Scand Audiol 1996;25:133-41.

6. Egan CA, Davies L, Halmagyi GM. Bilateral total deafness due to pontine haematoma. J Neurol Neurosurg Psychiatry 1996;61:628-31.

7. Smart CM, Giacino JT, Cullen T, Moreno DR, Hirsch J, Schiff ND, et al. A case of locked-in syndrome complicated by central deafness. Nat Clin Pract Neurol 2008; 4:448-53.

8. Goyal MK, Kumar G, Sahota PK. Reversible sensorineural hearing loss with normal brainstem auditory evoked 
potentials in pontine hemorrhage due to capillary telangiectasia. J Clin Neurosci 2010;17:1198-201.

9. Wada SI, Starr A. Generation of auditory brain stem responses (ABRs). II. Effects of surgical section of the trapezoid body on the ABR in guinea pigs and cat. Electroen- cephalogr Clin Neurophysiol 1983;56:340-51.

10. Krings T, Foltys H, Meister IG, Reul J. Hypertrophic olivary degeneration following pontine haemorrhage: hypertensive crisis or cavernous haemangioma bleeding? J Neurol Neurosurg Psychiatry 2003;74:797-9. 